\title{
Tuning the orbital angular momentum of high harmonics by manipulating the collinear photon channels in two-color high-harmonic generation
}

\author{
Zhe Wang $\odot,{ }^{1}$ Weiyi Hong, ${ }^{2, *}$ Feng Wang, ${ }^{1}$ and Qing Liao ${ }^{1, \dagger}$ \\ ${ }^{1}$ Hubei Key Laboratory of Optical Information and Pattern Recognition, Wuhan Institute of Technology, Wuhan 430205, China \\ ${ }^{2}$ Guangdong Provincial Key Laboratory of Nanophotonic Functional Materials and Devices, South China Normal University, \\ Guangzhou 510631, China
}

(Received 10 April 2020; accepted 9 September 2020; published 24 September 2020)

\begin{abstract}
The characteristics of the photon channels in the collinear two-color high-order harmonic generation (HHG) are investigated with the vortices. It is found that the yields of the photon channels sensitively depend on ratio of the intensities $I_{2 \omega} / I_{\omega}$. This sensitive dependence exhibits unique characteristics such as the suppression of the $n-\omega$ fundamental channel and the photon localization in the high-order channel. Therefore, one can adjust $I_{2 \omega} / I_{\omega}$ to select one dominant channel to generate and tune the harmonic vortices with well-defined orbital angular momentum (OAM). Our findings provide an effective way to generate extreme ultraviolet vortices or helical attosecond pulses with well-defined and controllable OAM.
\end{abstract}

DOI: 10.1103/PhysRevResearch.2.033482

\section{INTRODUCTION}

In the past years, remarkable advances have been achieved in the field of ultrafast optics [1-7]. Particularly, high-order harmonic generation (HHG) [8] is a topic of great interest because of its potential to produce coherent soft x-ray radiation and extreme ultraviolet (XUV) light. It has also been used for generation of isolated attosecond pulses. This allows for studying and controlling the ultrafast processes with unprecedented resolutions, such as pump-probe electronic dynamic measurements, time-resolved surface physics, attosecond streaking spectroscopy, and so forth [9]. The optimization of the properties of high-order harmonics has attracted much attention in recent years.

The configuration of the driving field imposes the characteristics of the HHG. Waveform synthesis schemes, more specifically the two-color scheme, have been regarded as very effective ways to control and optimize the harmonic properties [10-13]. Distinguished from one-color HHG, there are more than one photon channels participating in the HHG process. The identification and investigation of these photon channels can benefit the manipulation of harmonic process with the two-color field to optimize spatial and temporal properties of the high-order harmonics. For the noncollinear two-color HHG, Bertrand et al. [14] suggested that each photon channel in generating one harmonic has an individual divergence angle due to momentum and energy conservation, and therefore the

\footnotetext{
*Corresponding author: hongwy@m.scnu.edu.cn

$\dagger$ liaoqing@wit.edu.cn

Published by the American Physical Society under the terms of the Creative Commons Attribution 4.0 International license. Further distribution of this work must maintain attribution to the author(s) and the published article's title, journal citation, and DOI.
}

channels can be separated in space. In the collinear case, incommensurate frequency pulses are introduced to identify the number of photons in HHG process; i.e., the photon channels correspond to the generated noninteger high-order harmonics [15]. However, the low resolution of the spectrum and low intensity of the harmonics contributed by the weak channel may limit their observation and the further investigation. The characteristics of the collinear photon channel also possesses rich connotation of physics, which has not been clearly investigated and needs to be further studied.

Another topic for configuration of the driving field refers to the helical-phased beams, i.e., the so-called optical vortices. Besides the intrinsic spin angular momentum associated with the polarization, the spiral phase wind imprints additional orbital angular momentum (OAM) to the beam [16]. The generation of a laser pulse with OAM has been popular in recent years because the optical vortices have many potential applications in different fields. For the near-infrared and visible wavelengths, the laser pulse with OAM can easily be produced with a spatial light modulator (SLM) or designed photonic crystal fibers (PCF). When the wavelengths of such laser pulses extend to the XUV region, conventional methods can no longer work since the SLM or PCF can hardly be fabricated. Therefore, HHG is the candidate for the XUV pulse with OAM. As light-matter interaction is inherently connected with the exchange of momentum, OAM can be naturally transferred to high-order harmonics via HHG process [17-20]. This also gives an additional degree of complexity to shape the laser field for the engineering of the HHG beams. Recent studies showed a very promising perspective of the confluence of OAM and HHG. It has been proposed that the optical vortices can be combined with phase matching [21,22], spin constraint [23-25], and noncollinear scheme [26-28] in the manipulation of $\mathrm{HHG}$, in order to control the polarization and OAM of the XUV vortices or to obtain the helically shaped attosecond pulses. 


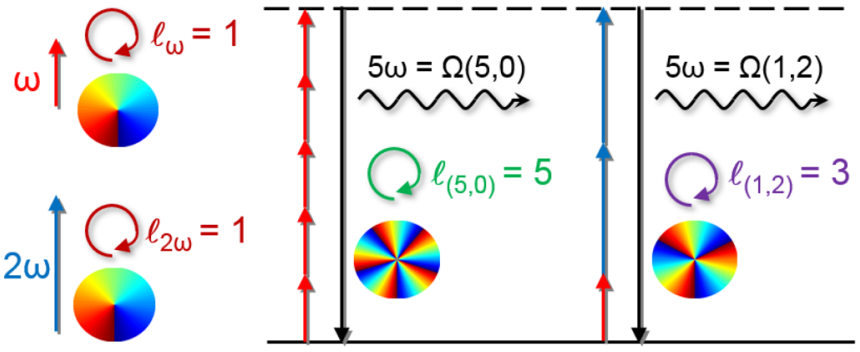

FIG. 1. Photon channel perspective of the two-color $\omega+2 \omega$ HHG driven by optical vortices. $n_{1}$ and $n_{2}$ in the description of the photon channel $\left(n_{1}, n_{2}\right)$ refer to the number of photons absorbed from the $\omega$ and $2 \omega$ fields.

In this paper, we focus on the characteristics of collinear photon channels in the two-color vortex HHG and propose a scheme to tune the OAM of the harmonics. We find that the $n-\omega$ fundamental photon channel for $n$ th-order harmonic is suppressed even if the ratio of the intensities $I_{2 \omega} / I_{\omega}$ is very small. More interestingly, the yields of the photon channels sensitively depend on the intensity ratio of the two-color fields, and photon localization in an individual channel can be achieved. In addition, referring to the model proposed by Li et al. [29], we present the qualitative explanation of our numerical observations. Note that the noncollinear scheme is also an effective and easy way to distinguish the photon channels. However, if one adopts the noncollinear scheme to produce the harmonics with well-defined OAM, the energy loss is unavoidable since the harmonics for different channels are separated in space and the spatial filtering is needed. For the spin-constraint method, the harmonic photons can only locate in the channels which satisfy the spin-constraint rule. In our scheme, it is found that one can adjust $I_{2 \omega} / I_{\omega}$ to select one dominant channel for harmonic generation. Therefore, the harmonic energy can be concentrated on a single channel without separation by other channels, and then the harmonics with well-defined OAM can be generated more efficiently. Besides, in our method every possible channel to generate one harmonic can be dominated, which indicates that the OAM of the harmonics can be tuned in an accessible way. Moreover, our scheme of tuning OAM by manipulating the collinear photon channels may combine with other traditional controlling methods in HHG (e.g., wave- form synthesis scheme, phasematching technique), which opens a promising perspective in the generation of helical attosecond or even isolated attosecond pulses with tunable and well-defined OAMs.

\section{IDENTIFICATION OF THE COLLINEAR PHOTON CHANNELS WITH OPTICAL VORTEX}

The sketch for the identification of the photon channels in the collinear two-color $\omega+2 \omega$ HHG is shown in Fig. 1. We consider a Laguerre-Gaussian (LG) beam [16] with the topological charge $\ell=1$ as the driving vortex beam, and therefore the OAM carried by each photon in the beam is 1 . Take the fifth-order harmonic as an example. There are two contributing channels $(5,0),(1,2)$ according to the conservation of energy and parity. They cannot be directly distinguished from the conventional harmonic spectrum due to the identity of the high-order harmonic photons. This identity is broken by introducing the optical vortices into the driving beams, since the harmonics originating from different channels may obtain different OAMs due to the OAM conservation. For the fifth-order harmonic, in detail, the corresponding OAMs of the channels $(5,0)$ and $(1,2)$ are 5 and 3 , respectively. This one-to-one correspondence between the photon channels and OAMs can be easily extended to other orders of HHG. Therefore, one can extract the information of the photon channels from the OAM composition of the high-order harmonics. The measurement for the phase profiles of the vortex harmonic beam can be achieved by observing the interference pattern with a reference Gaussian harmonic beam, and the difference in the number of fringes on either side of the interference pattern is the topology of the harmonic vortex [26,27]. In addition, the Fourier analysis of the interference pattern can also help the evaluation for the topology of the harmonic vortex.

\section{ANALYSIS OF THE HARMONIC YIELD IN THE TWO-COLOR SCHEME FROM THE PHOTON CHANNEL PERSPECTIVE}

With the method discussed above, we can directly obtain the characteristics of the harmonics from the photon channel perspective. It naturally raises an interesting question of how the harmonic yields of the photon channels depend on the configuration of the two-color driving field, especially the ratio between the two-color intensities. For this purpose, we perform a quantum simulation of argon driven by a two-color LG beam. The two-color field is synthesized by the linearly polarized 800- and 400-nm pulses. The pulse is assumed to have a trapezoidal envelope with one-cycle rise time, fourcycle plateau, and one-cycle fall time. The peak intensity of the $800-\mathrm{nm}$ pulse $I_{\omega}$ is $2 \times 10^{14} \mathrm{~W} / \mathrm{cm}^{2}$ ( $\omega$ is the frequency of the $800-n m$ pulse). The relative phase of the two-color field $\phi$ is 0 . The waists of the $\mathrm{LG}_{\omega}$ and $\mathrm{LG}_{2 \omega}$ beams are adjusted such that the radii of the intensity maxima in the focal plane coincide for both the $\omega$ and $2 \omega$ beams at $15 \mu \mathrm{m}$. We approximate the gas target by a two-dimensional thin layer of equally distributed atoms across the focus of the driving beam. The HHG signal is calculated by the Lewenstein model [30], and the ionization potential of argon atom is 0.5792 atomic units in the simulation. The propagation effect is also considered using the model discussed in Refs. [21,24]. It is worth mentioning that the HHG signals calculated with Lewenstein model contain the contributions from all the possible photon channels, which cannot be distinguished directly. For this purpose, the vortices have been introduced in the two-color scheme to distinguish the photon channel by detecting the OAM of the harmonic signals, due to the fact that there is a one-to-one correspondence between the photon channels and OAMs. The information of the photon channels can be obtained from the OAM composition of the high harmonics. We take the ninth-order harmonic as an example for analysis since it is the lowest order for more than three channels. As shown in Fig. 2(a), the enhancement of the ninth-order harmonic yield in the two-color field is clearly observed. Furthermore, the insets show that the phase profile of the ninth-order harmonic in the two-color field is different from that in one-color case, 



FIG. 2. (a) The angular-averaged harmonic spectra in the onecolor (blue line) and two-color (red line) field. (b) Variation of the photon channel yield with the increase of the weak $2 \omega$ field intensity for the ninth-order harmonic.

which corresponds to the changing of photon channels. There are three permitted photon channels contributing to the ninthorder harmonic: channels $(9,0),(5,2)$, and $(1,4)$. Figure 2(b) presents the yields of these three channels with the introduction of the weak $2 \omega$ field. We keep $I_{\omega}$ as constant and vary the ratio $r$ of the intensity $I_{2 \omega} / I_{\omega}$ from 0 to 0.1 for investigation. Note that the variation of the yields for the photon channels is caused by the introduction of the control field. When the intensity ratio $I_{2 \omega} / I_{\omega}$ is very small, some high-order photon channels may be not opened and the influence of intensity ratio on these channels is not obvious. As shown in Fig. 2(b), the yield of the channel $(1,4)$ is much lower than the other two channels with $r \leqslant 0.1$, so we focus on the channels $(9,0)$ and $(5,2)$. From Fig. 2(b), one can see an interesting phenomenon that the yield of the fundamental channel (9, 0 ) decays rapidly with the increase of $r$. The suppression of the fundamental channel can be qualitatively understood by the theoretical model discussed in Ref. [29], where a photon channel perspective of HHG is established by quantizing both the driving laser and high harmonics. According to the model in Ref. [29], the emission rate of the harmonic is proportional to $\sigma_{0}\left(n_{1}, n_{1}\right) C_{n_{1}+n_{2}}^{n_{1}}(1-P)^{n_{1}} P^{n_{2}}$, with $P=I_{2 \omega} /\left(I_{\omega}+I_{2 \omega}\right)$. The term $\sigma_{0}$ describes the characteristic structure of the high-order harmonic spectra and $\sigma_{0}$ is related to the total laser intensity. The term $C_{n_{1}+n_{2}}^{n_{1}}(1-P)^{n_{1}} P^{n_{2}}$ corresponds to the weight of the photon channel, which describes the channel competition of a specific harmonic. Specially, for the $(n, 0)$ fundamental channel, this term is expressed as $(1-P)^{n} P^{0}$. Then the yield of the fundamental photon would decrease with the increase of $I_{2 \omega}$ and this effect is more obvious for higher order harmonics. On the other hand, the yield of channel $(5,2)$ increases quickly with the increase of $r$. For the case of $r=0.1$, the channel $(5,2)$ predominates the harmonic yields and is more intense than the fundamental channel $(9,0)$ in the one-color field $(r=0)$. This implies that there are more photons participating in the HHG process from the high-order channel (5, 2) than the one-color fundamental channel, which explains in the photon-channel perspective why the two-color scheme can enhance the harmonic efficiency even if the intensity of the control field is very small. It is worth mentioning that we also made calculations of Fig. 2(b) with a fixed intensity $I_{\omega}+I_{2 \omega}$ of $2 \times 10^{14} \mathrm{~W} / \mathrm{cm}^{2}$, and the results are very similar with the fixed $I_{\omega}$ case. In addition, in Fig. 2(a) one can see that the third-order harmonic in the two-color field is nearly the same as that in the one-color field, as marked by the dashed rectangle. This is because there is only one photon channel (3, 0 ) contributing to the third-order harmonic generation. Using a two-color field cannot open new channels for more photons participating in harmonic process, and therefore the harmonic yield cannot be increased. This finding can further support our analysis.

\section{TUNING THE HARMONICS OAM BY MANIPULATING THE YIELDS OF THE PHOTON CHANNELS}

For the case of sufficiently high ratio $r$, more photon channels are opened: Both of the high-order channels $(5,2)$ and $(1,4)$ can effectively contribute to the ninth-order harmonic generation. In Fig. 3, we present the far-field transversal phase profiles [Figs. 3(a)-3(c)] and the photon channel distributions [Figs. 3(d)-3(f)] of the ninth-order harmonic as a function of the ratio $r$. We fix the total intensity of the $\omega+2 \omega$ field to $2 \times 10^{14} \mathrm{~W} / \mathrm{cm}^{2}$. Each OAM component can be extracted by the Fourier transform of the angular phase at the divergence angle with the maximum intensity. According to our calculations, the intensity of the ninth-order harmonic reaches maximum at the divergence angle of $8 \mathrm{mrad}$ in the far field. At this divergence angle, we perform the Fourier transform of the angular phase, and the intensities of the frequencies 9, 7 , and 5 in the Fourier spectrum correspond to the weights of three OAM components (or three photon channels) for the ninth-order harmonic. As shown in Fig. 3, the angular phase profile of the ninth-order harmonic presents a regularly periodical structure for $r=0.1$. The topological charge $\ell$ is 7 , which corresponds to the dominant channel $(5,2)$ as shown in Fig. 3(d). As $r$ is increased to 0.6, the intensities of the channel $(5,2)$ and channel $(1,4)$ are comparable. The interference of different OAMs from the two channels results in an irregular phase structure as shown in Fig. 3(b). When $r=1.5$, the intensity of the channel $(1,4)$ is dominant. Consequently, the ninth-order harmonic is generated with a well-defined OAM 

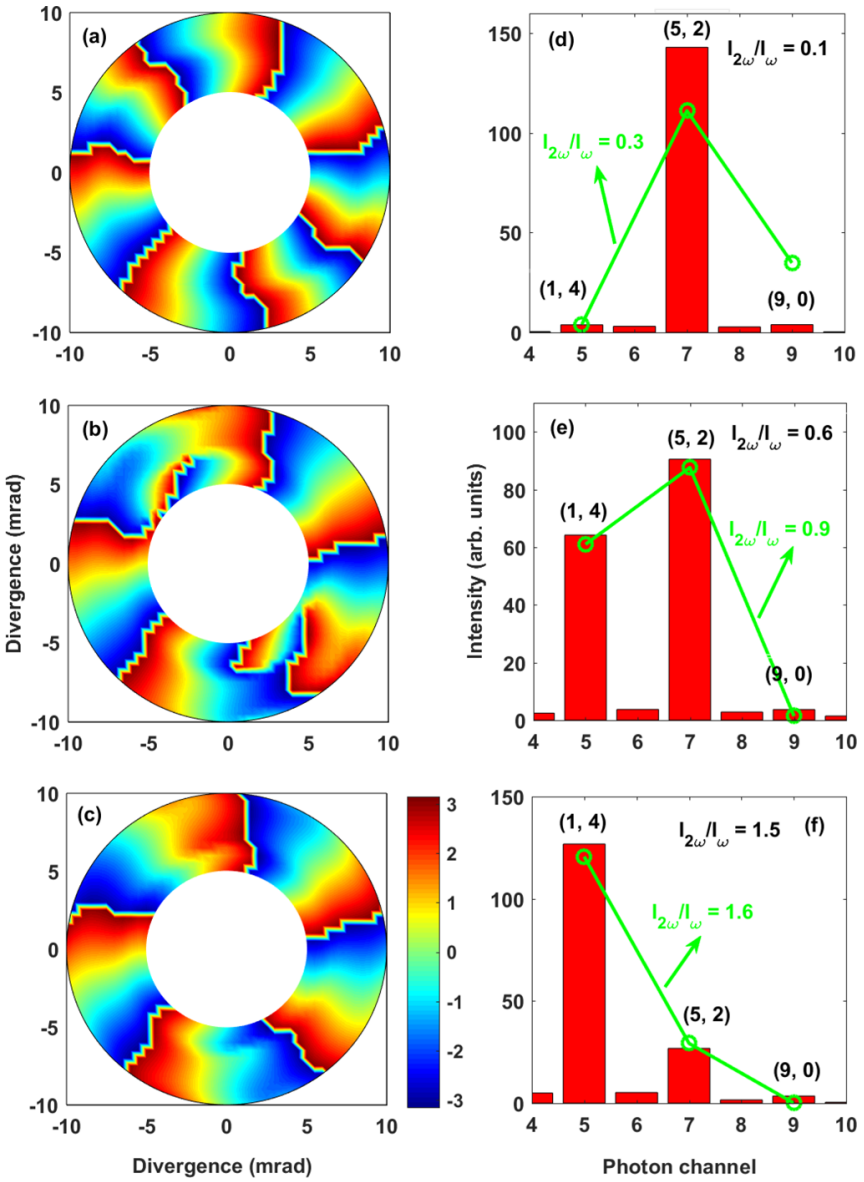

FIG. 3. Far-field transversal phase profiles [(a)-(c)] and photon channel distributions [(d)-(f)] of the ninth-order harmonic with the variation of the relative intensity $I_{2 \omega} / I_{\omega}$.

with $\ell=5$, which is shown in Fig. 3(c). The green curves present the photon channel yields calculated by Li's model. One can see that our results are qualitatively consistent with the predictions by Li's model: The competition of the photon channels is clearly observed, and the dominant channels can be tuned by adjusting $r$. In addition, we investigate the variation of the photon channels with different relative phases $\phi$ as shown in Fig. 4. The ratio $r$ is fixed to 0.1 . It is found that the phase profile maintains the structure except for a rotation. Therefore, we can conclude that the photon channel distributions do not vary with the relative phase of the two-color field.

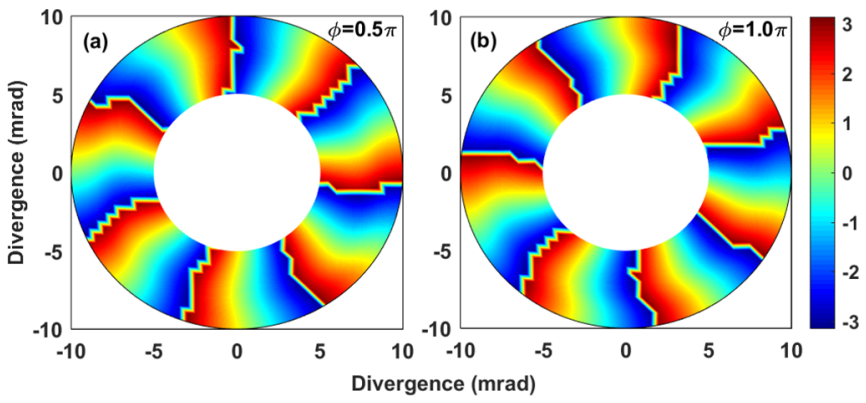

FIG. 4. Far-field phase profiles of the ninth-order harmonic with different relative phases of $\phi=0.5 \pi$ (a) and $\phi=1.0 \pi$ (b).
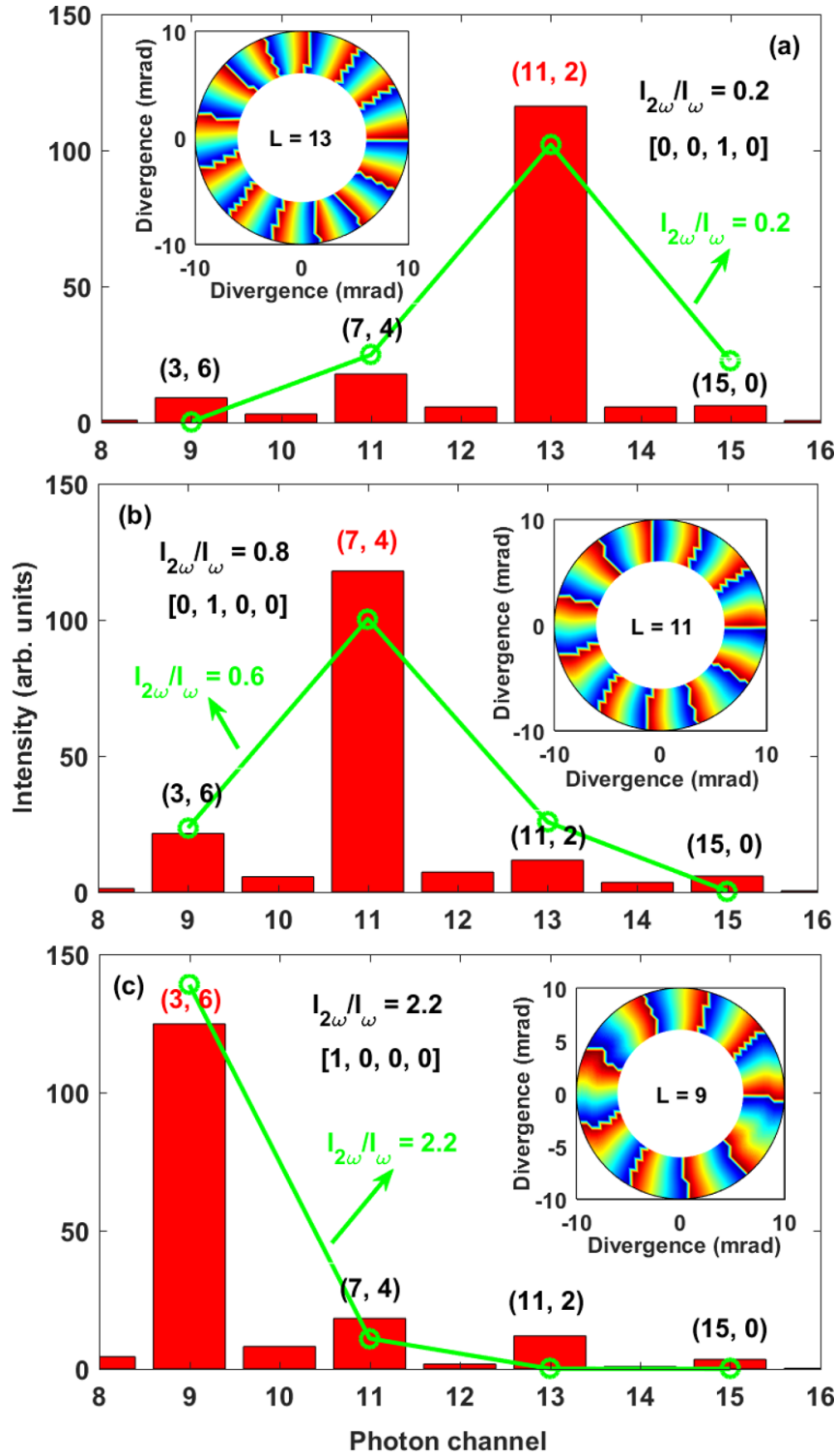

FIG. 5. Distributions of the photon channel for the 15th-order harmonic with different relative intensities. The insets are the farfield transversal phase profiles for each case.

In principle, the number of the permitted photon channels increases with the order of high harmonics. For instance, there are four possible photon channels for the 15th-order harmonic, i.e., $(15,0),(11,2),(7,4)$, and $(3,6)$, and the corresponding OAMs of these four channels are 15, 13, 11, and 9, respectively. Next, we would like to show that every photon channel can become dominant in sequence by varying the ratio $r$. The result is shown in Fig. 5. It is clear that our results are in good agreement with the predictions of Li's model. The fundamental channel $(15,0)$ is always suppressed, and the photons can be localized in other three high-order channels for the special value of $r$. In these cases, the OAM of the 15 th-order harmonic can be well defined with $\ell=13,11$, and 9, as shown by the insets of Fig. 5. This result provides an accessible way to produce XUV vortices with controllable OAM by switching the photon channels. Besides, the photon localization here may also benefit the realization of optical 
quantum encoding and communication with the strong field effect. For example, we consider the photon channels with the intensity lower than 30 (arbitrary units) are closed. The open and closed status of the channel are set to " 1 " and " 0 ." The values $\mathrm{a}, \mathrm{b}, \mathrm{c}$, and $\mathrm{d}$ in the binary code $[\mathrm{a}, \mathrm{b}, \mathrm{c}, \mathrm{d}]$ correspond to the status of the channels $(3,6),(7,4),(11,2)$, and $(15,0)$. Then the corresponding binary codes in Figs. 5(a), 5(b), and $5(\mathrm{c})$ are $[0,0,1,0],[0,1,0,0]$, and $[1,0,0,0]$, respectively.

\section{CONCLUSION}

In conclusion, we investigate the characteristics of the photon channels in the two-color $(\omega+2 \omega)$ collinear vortex HHG, and find that the yields of the photon channels sensitively depend on the ratio $r$ of $I_{2 \omega} / I_{\omega}$. The $n-\omega$ fundamental photon channel for $n$ th-order harmonic is suppressed even if $r$ is very small. By adjusting $r$, the photon localization in an individual high-order channel can be achieved, which allows the OAM tuning of the harmonics. These findings provide an effective way to generate XUV vortices or helical attosecond pulses with tunable and well-defined OAM and may become a candidate in optical quantum encoding using the strong field effect.

\section{ACKNOWLEDGMENTS}

This work was supported by the National Natural Science Foundation of China under Grants No. 11874019, No. 11747041, and No. 11674257, the Natural Science Foundation of Guangdong Province under Grant No. 2019A1515011172, and the Natural Science Foundation of Hubei Province under Grant No. 2017CFB150.
[1] P. B. Corkum and F. Krausz, Nat. Phys. 3, 381 (2007).

[2] E. Goulielmakis, M. Schultze, M. Hofstetter, V. S. Yakovlev, J. Gagnon, M. Uiberacker, A. L. Aquila, E. M. Gullikson, D. T. Attwood, R. Kienberger, F. Krausz, and U. Kleineberg, Science 320, 1614 (2008).

[3] J. Miao, T. Ishikawa, I. K. Robinson, and M. M. Murnane, Science 348, 530 (2015).

[4] Z. Tao, C. Chen, T. Szilvási, M. Keller, M. Mavrikakis, H. Kapteyn, and M. Murnane, Science 353, 62 (2016).

[5] L. He, P. Lan, A.-T. Le, B. Wang, B. Wang, X. Zhu, P. Lu, and C. D. Lin, Phys. Rev. Lett. 121, 163201 (2018).

[6] J. Tan, Y. Zhou, M. He, Y. Chen, Q. Ke, J. Liang, X. Zhu, M. Li, and P. Lu, Phys. Rev. Lett. 121, 253203 (2018).

[7] K. Liu, S. Luo, M. Li, Y. Li, Y. Feng, B. Du, Y. Zhou, P. Lu, and I. Barth, Phys. Rev. Lett. 122, 053202 (2019).

[8] X. F. Li, A. L'Huillier, M. Ferray, L. A. Lompré, and G. Mainfray, Phys. Rev. A 39, 5751 (1989).

[9] F. Krausz and M. Ivanov, Rev. Mod. Phys. 81, 163 (2009).

[10] J. Mauritsson, P. Johnsson, E. Gustafsson, A. L'Huillier, K. J. Schafer, and M. B. Gaarde, Phys. Rev. Lett. 97, 013001 (2006).

[11] P. Lan, P. Lu, W. Cao, Y. Li, and X. Wang, Phys. Rev. A 76, 011402(R) (2007).

[12] Z. Zeng, Y. Cheng, X. Song, R. Li, and Z. Xu, Phys. Rev. Lett. 98, 203901 (2007).

[13] E. J. Takahashi, P. Lan, O. D. Mücke, Y. Nabekawa, and K. Midorikawa, Phys. Rev. Lett. 104, 233901 (2010).

[14] J. B. Bertrand, H. J. Wörner, H.-C. Bandulet, É. Bisson, M. Spanner, J.-C. Kieffer, D. M. Villeneuve, and P. B. Corkum, Phys. Rev. Lett. 106, 023001 (2011).

[15] A. Fleischer, O. Kfir, T. Diskin, P. Sidorenko, and O. Cohen, Nat. Photonics 8, 543 (2014).

[16] L. Allen, M. W. Beijersbergen, R. J. C. Spreeuw, and J. P. Woerdman, Phys. Rev. A 45, 8185 (1992).

[17] M. Zürch, C. Kern, P. Hansinger, A. Dreischuh, and C. Spielmann, Nat. Phys. 8, 743 (2012).
[18] C. Hernández-García, A. Picón, J. San Román, and L. Plaja, Phys. Rev. Lett. 111, 083602 (2013).

[19] R. Géneaux, A. Camper, T. Auguste, O. Gobert, J. Caillat, R. Taïeb, and T. Ruchon, Nat. Commun. 7, 12583 (2016).

[20] L. Rego, J. S. Román, A. Picón, L. Plaja, and C. HernándezGarcía, Phys. Rev. Lett. 117, 163202 (2016).

[21] C. Hernández-García, J. San Román, L. Plaja, and A. Picón, New J. Phys. 17, 093029 (2015).

[22] R. Géneaux, C. Chappuis, T. Auguste, S. Beaulieu, T. T Gorman, F. Lepetit, L. F. DiMauro, and T. Ruchon, Phys. Rev. A 95, 051801(R) (2017).

[23] K. M. Dorney, J. L. Ellis, C. Hernández-García, D. D. Hickstein, C. A. Mancuso, N. Brooks, T. Fan, G. Fan, D. Zusin, C. Gentry, P. Grychtol, H. C. Kapteyn, and M. M. Murnane, Phys. Rev. Lett. 119, 063201 (2017).

[24] W. Paufler, B. Böning, and S. Fritzsche, Phys. Rev. A 98, 011401(R) (2018).

[25] K. M. Dorney, L. Rego, N. J. Brooks, J. San Román, C.-T. Liao, J. L. Ellis, D. Zusin, C. Gentry, Q. L. Nguyen, J. M. Shaw, A. Picón, L. Plaja, H. C. Kapteyn, M. M. Murnane, and C. Hernández-García, Nat. Photonics 13, 123 (2019).

[26] G. Gariepy, J. Leach, K. T. Kim, T. J. Hammond, E. Frumker, R. W. Boyd, and P. B. Corkum, Phys. Rev. Lett. 113, 153901 (2014).

[27] F. Kong, C. Zhang, F. Bouchard, Z. Li, G. G. Brown, D. H. Ko, T. J. Hammond, L. Arissian, R. W. Boyd, E. Karimi, and P. B. Corkum, Nat. Commun. 8, 14970 (2017).

[28] D. Gauthier, P. R. Ribič, G. Adhikary, A. Camper, C. Chappuis, R. Cucini, L. F. DiMauro, G. Dovillaire, F. Frassetto, R. Géneaux, P. Miotti, L. Poletto, B. Ressel, C. Spezzani, M. Stupar, T. Ruchon, and G. D. Ninno, Nat. Commun. 8, 14971 (2017).

[29] L. Li, P. Lan, L. He, X. Zhu, J. Chen, and P. Lu, Phys. Rev. Lett. 120, 223203 (2018).

[30] M. Lewenstein, P. Balcou, M. Yu. Ivanov, A. L'Huillier, and P. B. Corkum, Phys. Rev. A 49, 2117 (1994). 